\title{
A Drosophila Temperature-Sensitive Seizure Mutant in Phosphoglycerate Kinase Disrupts ATP Generation and Alters Synaptic Function
}

\author{
Ping Wang, ${ }^{1 \star}$ Sudipta Saraswati, ${ }^{1 *}$ Zhuo Guan, ${ }^{1}$ Carol J. Watkins, ${ }^{2}$ Richard J. Wurtman, ${ }^{2}$ and J. Troy Littleton ${ }^{1,2}$ \\ ${ }^{1}$ The Picower Center for Learning and Memory and Department of Biology, and ${ }^{2}$ Department of Brain and Cognitive Sciences, Massachusetts Institute of \\ Technology, Cambridge, Massachusetts 02139
}

\begin{abstract}
A novel paralytic mutant, nubian, was identified in a behavioral screen for conditional temperature-sensitive seizure mutants in Drosophila melanogaster. nubian mutants display reduced lifespan, abnormal motor behavior, altered synaptic structure, and defective neurotransmitter release. The nubian mutant disrupts phosphoglycerate kinase (PGK), an enzyme required for ATP generation in the terminal stage of the glycolytic pathway. Consistent with altered ATP generation in nubian animals, brain extracts show a threefold reduction in resting ATP levels compared with controls. Microarray analysis of nubian mutants reveals altered transcription of genes implicated in glucose and lipid metabolism. Disruption of ATP generation in nubian animals is accompanied by temperature-dependent defects in neuronal activity, with initial seizure activity, followed by an activity-dependent loss of synaptic transmission. nubian mutants also display structural defects at the synapse, with larger varicosity size but normal varicosity number, indicating that these synaptic parameters are regulated independently. Both exocytotic (NSF) and endocytotic (dynamin) ATPase/GTPase activity are required for normal synaptic transmission. Biochemical and physiological analyses indicate that synaptic defects in nubian animals are secondary to defective endocytosis, suggesting that endocytotic pathways may be generally more sensitive to altered ATP levels than those used for exocytosis. Alterations in ATP metabolism likely disrupt similar pathways in humans, because PGK deficiency is associated with mental retardation, seizures, and exercise intolerance. Given the behavioral similarities between disruptions of PGK function in Drosophila and humans, the analysis of nubian animals may reveal conserved neuronal responses associated with altered ATP generation within the brain.
\end{abstract}

Key words: epilepsy; synaptic transmission; glycolysis; Drosophila; endocytosis; seizure; microarray

\section{Introduction}

The fundamental features of synaptic transmission have been well characterized at the electrophysiological level and were expounded on originally by Katz (1969) when he introduced the "calcium hypothesis" of neurotransmitter release. Within this basic framework, neurons show a remarkable ability to adapt to changes in synaptic input via homeostatic compensation mechanisms that maintain neurotransmission within appropriate levels of excitation (Turrigiano, 1999; Davis and Bezprozvanny, 2001). Neurological diseases can disrupt this balance and lead to dramatic changes in neuronal activity. Such alterations in neuronal signaling have important roles in pathological states such as

\footnotetext{
Received 0ct. 8, 2003; revised March 22, 2004; accepted March 23, 2004.

This work was supported by National Institutes of Health Grants NS40296, NS43244, MH28783, the Human Frontiers Science Program Organization, the Packard Foundation, and the Searle Scholars Program. J.T.L. is an Alfred P. Sloan Research Fellow. We thank Enrico Montana for helpful discussions about this manuscript and Tom Lloyd for fly stocks and helpful advice. The $8 \mathrm{C} 3$ antibody developed by Seymour Benzer was obtained from the Developmental Studies Hybridoma Bank developed under the auspices of the National Institute of Child Health and Human Development and maintained by the Department of Biological Sciences, University of lowa, lowa City, IA.

*P.W. and S.S. contributed equally to this work.

Correspondence should be addressed to J. Troy Littleton, The Picower Center for Learning and Memory, Massachusetts Institute of Technology, E18-672, 50 Ames Street, Cambridge, MA 02139. E-mail: troy@mit.edu. DOI:10.1523/JNEUROSCI.0542-04.2004

Copyright $\odot 2004$ Society for Neuroscience $\quad$ 0270-6474/04/244518-12\$15.00/0
}

epilepsy, a recurrent seizure syndrome caused by abnormal synchronized neuronal firing. Over 100 known human Mendelian diseases include epilepsy as part of the neurological manifestation, although only a few genes contributing to distinct epilepsy syndromes in humans have been identified (Gardiner, 1999). So that this lack of molecular insights into human epilepsy might be circumvented, genetic approaches have been used in model organisms to define neuronal pathways associated with seizure activity. To this end, genetic manipulations in the mouse have identified several genes that cause epilepsy-like syndromes when mutated (Puranam and McNamara, 1999). More recently, genetic approaches in Drosophila have been used to identify seizure mutants that activate conserved signaling pathways in response to excessive neuronal activity (Kuebler et al., 2001; Hoeffer et al., 2003).

To elucidate further the mechanisms that lead to seizures, we performed temperature-sensitive (TS) behavioral screens for seizure-inducing mutants in Drosophila. TS paralytic mutations have been an important tool in the genetic dissection of membrane excitability, identifying essential proteins required for membrane excitability, endocytosis, and exocytosis (for review, see Ganetzky and $\mathrm{Wu}, 1986)$. Forward genetic TS screens allow mutations in proteins important in neuronal signaling to be se- 
lected on the basis of behavioral paralysis, without any previous knowledge of the products involved. They also provide an inducible mutagenesis approach, allowing one to remove or reduce the function of the protein at any stage of development. We have used this strategy to identify the nubian locus, a mutation that leads to inducible seizures, followed by a complete block in synaptic transmission. The nubian mutant disrupts phosphoglycerate kinase (PGK), an enzyme required for ATP generation in the terminal stage of the glycolytic pathway. We demonstrate that nubian mutants block synaptic transmission in an activitydependent manner via disruption of synaptic vesicle endocytosis. PGK deficiency in humans has been identified as an X-linked metabolic myopathy causing exercise intolerance (DiMauro et al., 1983). Recently, PGK deficiency has been shown to manifest in a variety of symptoms, depending on the severity of enzyme dysfunction (Tsujino et al., 1995). These include mental retardation, seizures, and myopathy, indicating the importance of energy metabolism in the acute function of the nervous and muscular systems. Much effort has gone into characterizing energy use in muscle, in which metabolic myopathies represent major clinical syndromes (Tein, 1996). However, the effect of reduced ATP levels on brain function, as occur during stroke, oxygen deprivation, and inherited metabolic disorders, has been more difficult to define. Here we describe our analysis of the in vivo consequences on neuronal physiology and synaptic structure of both acute and chronic reductions in ATP generation in the $n u$ bian mutant.

\section{Materials and Methods}

Drosophila genetics. Drosophila melanogaster were cultured on standard medium at $22^{\circ} \mathrm{C}$. Temperature-sensitive (TS) behavioral mutants on the second chromosome were generated by feeding 1 - to 2-d-old isogenized cn males with ethyl methanesulfonate (EMS) for $12 \mathrm{hr}$ and mating to marked balancer chromosomes via standard techniques (Lewis and Bacher, 1968). Homozygous F3 males were collected after F2 heterozygous sibling matings and were tested for TS paralysis in a $38^{\circ} \mathrm{C}$ water bath. $p g k^{\text {nubian }}$ was mapped to $2-5$ by recombination with $S p J L P$-marked chromosomes.

Sequencing. pgk $k^{\text {nubian }}$ and CS genomic DNA was used as a template to PCR-amplify a genomic fragment spanning the complete open reading frame (ORF) of both $p g k$ and CG9961. High-fidelity Taq (Takara USA, New York, NY) was used to minimize PCR errors. Sequencing primers encompassing the ORF were used to sequence in duplicate from the two templates. PCR products were purified with the QIAquick PCR Purification Kit (Qiagen, Valencia, CA) and sequenced by MWG Biotech (Ebersberg, Germany). The primers that were used included the following: pgk-5' -(GGAATCAGTGGTATCGCAATCCGGAG), pgk-3' -(CGTCGTGGTTTGTGCTTACAAGGTAAAC), CG9961-5' -(CCTCATCGTGCATTGTATTTTCAGTC), and CG9961-3' -(GATACATGTACATATAAGTGGAATAAC).

Sequences were aligned and compared by using commercially available software.

ATP analysis. Thirty CS and $p g k^{\text {nubian }}$ adults ( 15 males $/ 15$ females) aged 3-5 d were collected at room temperature and transferred to $15 \mathrm{ml}$ Falcon tubes. Flies of the indicated genotype were frozen in liquid nitrogen and vortexed to separate heads from bodies. Isolated heads from each genotype were homogenized in cold $80 \%$ methanol, centrifuged, and lyophilized. The extract was redissolved in water and centrifuged before the supernatants were frozen. Thawed samples were recentrifuged, and nucleotide analysis was performed via HPLC with a modified Simmonds method (Simmonds et al., 1988) on a Beckman System Gold (Beckman Coulter, Fullerton, CA). Separation was performed on a $5 \mu \mathrm{m}$ Hypersil APS-2 $\left(\mathrm{NH}_{2}\right)$ column $(250 \times 4.6 \mathrm{~mm}$; Alltech, Nicholasville, $\mathrm{KY})$ equilibrated with $2.5 \mathrm{mM} \mathrm{H}_{3} \mathrm{PO}_{4}$, $\mathrm{pH} 2.8$, switched via a 1 min linear gradient to $500 \mathrm{~mm} \mathrm{NaH}_{2} \mathrm{PO}_{4}$ at $\mathrm{pH} 4.6$, and held for $20 \mathrm{~min}$ (flow rate, $1 \mathrm{ml} / \mathrm{min}$; $37^{\circ} \mathrm{C}$ ). The nucleotides were quantified by on-line detection at $259 \mathrm{~nm}$, with ADP eluting at $9.4 \mathrm{~min}$ and ATP at 12 min under these conditions. Triplicate measurements were made for each sample. In addition, three independent assays were performed with different batches of control and $p g k^{\text {nubian }}$ animals. Results were averaged and presented as the ADP/ATP ratio \pm STD.

Microarray analysis. Microarray analysis was performed with Affymetrix Drosophila Genechips (Santa Clara, CA), using biotinylated cRNA and using the laboratory methods described in the Affymetrix Genechip Expression manual. CS and $p g k^{\text {nubian }}$ males were allowed to age 3-4 d after eclosion at room temperature. All flies were killed between 2 and 4 P.M. to reduce any circadian-dependent transcriptional changes. Total RNA was extracted from 600 pooled fly heads via the Trizol method (Invitrogen, San Diego, CA). Three independent RNA samples were prepared from each genotype. Poly(A) RNA was isolated, and $2 \mathrm{mg}$ was used to produce cDNA via the Superscript Double-Stranded cDNA Synthesis kit (Invitrogen). cRNA was synthesized with biotinylated NTPs (Invitrogen) and fragmented to produce 35-200 bp fragments for gene chip hybridization. Affymetrix high-density oligonucleotide arrays were probed, hybridized, stained, and washed in the Massachusetts Institute of Technology Biopolymers Facility according to the manufacturer's instructions. Microarray analysis was performed with Microarray Suite version 5 and Data Mining Tool version 3 statistics-based analysis software (Affymetrix). In addition to statistical analysis by Affymetrix, Student's $t$ tests and Mann-Whitney $U$ tests were performed to compare absolute signals from CS and $p g k^{\text {nubian }}$. We required both statistically significant $(p<0.05)$ transcriptional changes and a detection call by the Affymetrix software ( $\mathrm{P}$ or $\mathrm{M}$ ) for at least one of the two genotypes before the gene was accepted as differentially regulated.

Electrophysiology analysis. Electrophysiological analysis of wandering stage third instar larva was performed in Drosophila saline [containing (in mM): $70 \mathrm{NaCl}, 5 \mathrm{KCl}, 4 \mathrm{MgCl}_{2}, 0.2 \mathrm{CaCl}_{2}, 10 \mathrm{NaHCO}_{3}, 5$ Trehalose, 115 sucrose, 5 HEPES-NaOH, pH 7.2, modified from HL3 (Stewart et al., 1994)], using an Axoclamp 2B amplifier (Axon Instruments, Foster City, $\mathrm{CA}$ ) at $22^{\circ} \mathrm{C}$. Elevated temperature recordings at $36^{\circ} \mathrm{C}$ were performed at muscle fiber 6/7 of segments A3-A5 under current clamp. During high temperature experiments the preparations were maintained at $36^{\circ} \mathrm{C}$ for 3 min before stimulation. For recording the output of the central pattern generator in third instar larvae, we left motor neurons intact with the ventral nerve cord and bathed the preparation in saline containing 1.5 $\mathrm{mm}$ extracellular calcium. In adults, extracellular field potentials were recorded by placing a sharp glass electrode near the longitudinal flight muscles after piercing the cuticle, with a reference electrode in the fly head. Temperature shifts were performed by heating mounting clay encompassing the fly to the desired temperature with a Peltier heating device. Electroretinograms were performed as previously described (Littleton et al., 1998).

Morphological analysis. Immunostaining was performed on third instar larvae at wandering stage after rearing at $22^{\circ} \mathrm{C}$. Larvae were dissected in Drosophila saline and fixed in 4\% formaldehyde in HL3 (Stewart et al., 1994) for 45 min. DSYT2 against synaptotagmin I (Littleton et al., 1993) was used at 1:1000. Rhodamine-phalloidin was used to label muscle actin for muscle surface area measurements. Immunoreactive proteins were visualized on a Zeiss Pascal Confocal (Oberkochen, Germany) by using fluorescent secondary antibodies (Molecular Probes, Eugene, OR; Chemicon, Temecula, CA; Jackson ImmunoResearch, West Grove, PA). We quantified larval neuromuscular junctions (NMJs) at muscles 6/7 in segments A3-A5. Bouton number was scored by counting each distinct spherical anti-SYT-stained varicosity along motor terminals. Bouton diameter was calculated by measuring the diameter of the five largest type $1 \mathrm{~B}$ boutons at each muscle $6 / 7$ synapse in segments A3-A5 by using $63 \times$ images and LSM 5 Pascal Analysis software. Muscles were photographed at $10 \times$, and muscle surface area (in $\mu \mathrm{m}^{2}$ ) was computed on line with LSM 5 Pascal Analysis software. All error measurements are \pm SEM unless indicated.

Larval locomotion analysis. To quantify larval locomotion, we collected late third instar larvae grown at room temperature, washed them gently with distilled water, and placed them on a flat layer of $0.7 \%$ agarose maintained on an evenly illuminated light box (Wang et al., 1997; Saraswati et al., 2004). After observing the first locomotor cycle, we ini- 
tiated video recording of locomotion with a video camera (Canon XL1S) attached to a $16 \times$ zoom lens. The recording field was $27 \mathrm{~cm}^{2}$, and each larva was recorded for at least $2 \mathrm{~min}$. Larval images were digitized at 2 frames/sec on a Macintosh G4 computer with an RTMac frame grabber. Digital movies were analyzed by Dynamic Image Analysis System (DIAS 3.2, Solltech, Oakdale, IA) motion analysis software (Wang et al., 1997). The DIAS software outlines the larva on each frame of a digital movie. The outlines were stacked to recreate locomotion patterns. Discontinuities in crawling pattern attributable to stage movement were corrected by joining disconnected paths. Speed was calculated by averaging the speed of the centroid (the center of the area of a larval perimeter) for each frame of the digital movie, using the central difference method (Soll, 1995). Larval speed for each frame $n$ was determined by dividing the length of a cord connecting the centroids at frame $(n-1)$ with that at $(n+1)$ and dividing by $2 t$, where $t$ is the time interval between analyzed frames (Wang et al., 1997). Direction changes representing the change of angle of the centroid for each frame were computed by using the central difference method (Soll, 1995). The frame-by-frame data for direction change were used to calculate the time spent in locomotion. Periods of locomotion were defined as a crawling segment consisting of five or more consecutive frames in which direction change was $<20^{\circ}$ for each frame when digitized at 2 frames/sec (Wang et al., 1997, 2002; Saraswati et al., 2004). Pausing was defined as frames in which direction change per frame was $>20^{\circ}$ at 2 frames/sec resolution. The frequency of locomotor cycles was calculated by counting the number of cycles and measuring their duration in a crawling segment that consisted of at least five consecutive cycles directly from video recordings (Saraswati et al., 2004). For determining cycle frequency, we selected crawling segments in which the larva was traveling at its maximum speed. Distance traveled was measured by analyzing the tracks created by a crawling larva on the agarose surface during a period of $2 \mathrm{~min}$ and then quantifying the number of 3 $\mathrm{mm}$ grid squares that were traversed.

Adult behavioral analysis. Adult climbing assays were performed as described previously (Richards et al., 1996). Briefly, flies were aged 4-5d, and the time required to climb $5 \mathrm{~cm}$ in a graduated plastic vial was recorded, with a cutoff of $5 \mathrm{~min}$. Spontaneous locomotion was measured in 4- to 5-d-old flies after introduction into a cylindrical plastic chamber (diameter, $8 \mathrm{~mm}$; height, $3 \mathrm{~mm}$ ) and quantifying the number of times individual flies spontaneously crossed a line bisecting the chamber within a 4 min period (Joiner and Griffith, 1999). Drosophila viability assays were performed by quantifying lifespan of $100 \mathrm{CS}$ and $p g k^{\text {nubian }}$ virgin male flies aged in a $28^{\circ} \mathrm{C}$ incubator. Ten flies of each genotype were placed in individual food vials transferred daily in a $28^{\circ} \mathrm{C}$ incubator. Lethality was quantified daily for each genotype.

Preparation of 75 complexes. Flies of the desired genotype were frozen in liquid nitrogen and vortexed; equal numbers of heads (10) for each genotype were homogenized in $50 \mu$ l of SDS sample buffer on ice. The samples were centrifuged briefly to pellet cuticle, and $20 \mu$ lof the supernatant was resuspended in $30 \mu \mathrm{l}$ of SDS sample buffer. Samples were loaded onto discontinuous $9 \% / 15 \%$ SDS-PAGE gels without boiling and separated at $15 \mathrm{mV} / \mathrm{gel}$. After transfer the blots were probed with antisyntaxin monoclonal antibody $8 \mathrm{C} 3$ at 1:1000 dilution. Immunoreactive bands were visualized via ECL (Pierce, Rockford, IL).

\section{Results}

To identify mutations that disrupt synaptic function and cause seizures, we conducted unbiased behavioral screens in Drosophila for EMS-induced TS mutations that cause paralysis at $38^{\circ} \mathrm{C}$. The goal of these screens is to generate thermolabile proteins or uncover thermosensitive processes important in neuronal signaling pathways that alter synaptic transmission when disrupted (Suzuki et al., 1971; Siddiqi and Benzer, 1976; Atkinson et al., 1991; Zinsmaier et al., 1994; Andrews et al., 1996; Koenig and Ikeda, 1996; Titus et al., 1997; Littleton et al., 1998; Yuan and Ganetzky, 1999; Kawasaki et al., 2000; Rao et al., 2001; Rikhy et al., 2003). Potential TS mutations on the second chromosome were generated by EMS mutagenesis in $\mathrm{F} 3$ screens and tested for paralysis in preheated glass vials in a $38^{\circ} \mathrm{C}$ water bath. Flies demonstrating abnormal motor behavior and paralysis were maintained as lab stocks, providing the starting point for a systematic analysis of synaptic function in a group of behavioral mutants enriched for those that disrupt neuronal signaling. To date, 27 complementation groups on the $\mathrm{X}$, second, and third chromosomes have been identified in large-scale screening efforts of EMS-induced TS mutants. As a cohesive strategy for cataloging the new TS complementation groups we identified, we named the mutants after cultural dances, providing a loose connection to the abnormal movements elicited at $38^{\circ} \mathrm{C}$ in the flies. Here we describe the characterization of a novel second-chromosome TS mutant identified in our screen, which we termed nubian (named after a dance originating from southern Egypt).

After isolation the TS mutants were subjected to two additional screening procedures to identify electrophysiological defects in neuronal signaling at $38^{\circ} \mathrm{C}$. First, the mutants were analyzed for temperature-induced seizure activity by screening for recurrent spontaneous neural activity in the adult giant fiber system at the nonpermissive temperature (Fig. $1 A-C$ ). The giant fiber flight circuit is critical for escape responses and flight initiation and includes both chemical and electrical synapses. The pathway can be activated via stimulation of the brain, and extracellular recordings can be made from the dorsal longitudinal flight muscles (DLMs). Wild-type Canton-S (CS) Drosophila display very little spontaneous activity when the temperature is raised to $38^{\circ} \mathrm{C}$ (Fig. $1 \mathrm{~A}$ ). In contrast, the nubian mutant exhibited robust spontaneous action potential spiking in the DLM flight muscles at $38^{\circ} \mathrm{C}$ (Fig. $\left.1 \mathrm{~B}, \mathrm{C}\right)$. The activation of the DLM flight muscles reflects the firing rate of the innervating motor neurons. The onset of seizure activity in nubian was observed immediately when animals were shifted to $38^{\circ} \mathrm{C}$ but rapidly subsided within minutes when maintained at $38^{\circ} \mathrm{C}$.

To characterize the nubian mutant further, we screened for electrophysiological defects in synaptic transmission in the visual system at permissive $\left(21^{\circ} \mathrm{C}\right)$ and nonpermissive temperatures $\left(38^{\circ} \mathrm{C}\right)$. The electroretinogram (ERG) is an extracellular recording from the Drosophila eye that measures light-induced depolarization of photoreceptors and synaptic activation of secondorder neurons in the visual system (Hotta and Benzer, 1969; Pak et al., 1969). The synaptic events occur at the onset and termination of a light pulse and are represented by the on- and offtransients of the ERG. Several known TS mutations that block synaptic transmission have been shown to lose on-/off-transients in a temperature-dependent manner, including $s y x^{3-69}$ and comt (Littleton et al., 1998), SNAP-25 ${ }^{T S}$ (Rao et al., 2001), csp (Zinsmaier et al., 1994), and shi (Siddiqi and Benzer, 1976). In a similar manner, nubian mutants lost the on-/off-transients at $38^{\circ} \mathrm{C}$ (Fig. $1 D, E)$, suggesting a defect in synaptic transmission in the visual pathway. The time course of the loss of on/offs in nubian animals resembled both $\operatorname{comt}^{S T 17}$ mutants that disrupt the ATPase NSF and $s h{ }^{T S 1}$ mutants that disrupt the GTPase dynamin (Siddiqi and Benzer, 1976; Littleton et al., 1998), requiring several minutes of exposure to $38^{\circ} \mathrm{C}$ before the transients disappeared. In addition, similar to $c o m t^{S T 17}$ and $s h i^{T S 1}$, the recovery of on-/off-transients on return to the permissive temperature in nubian was slow, requiring $\sim 30 \mathrm{~min}$ to recover from a $4 \mathrm{~min}, 38^{\circ} \mathrm{C}$ heat pulse. However, in contrast to $s h i^{T S 1}$ and comt $t^{S T 17}$ mutants, nubian animals also showed a slow recovery of photoreceptor repolarization (Fig. $1 E$, arrowhead), suggesting that membrane excitability is also defective in photoreceptors. The temperature-dependent loss of synaptic transmission in nubian animals prompted us to clone and characterize the affected locus.

To understand how the physiological defects manifested in 


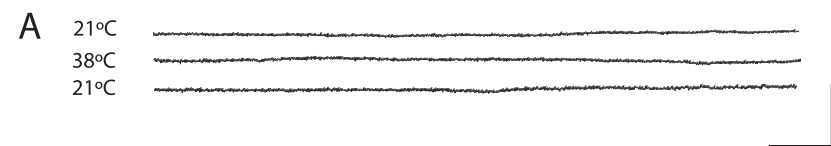

B $21^{\circ} \mathrm{C}$ $38^{\circ} \mathrm{C}$

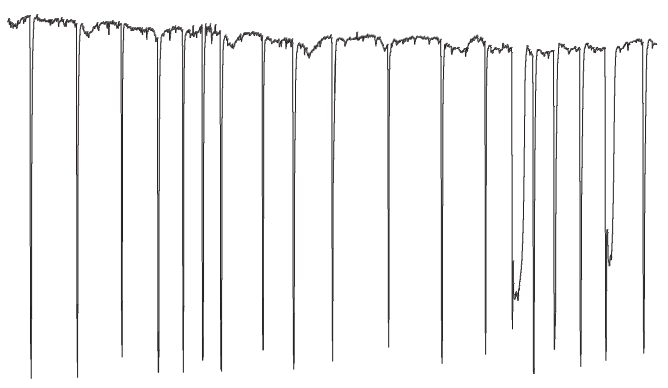

$21^{\circ} \mathrm{C}$

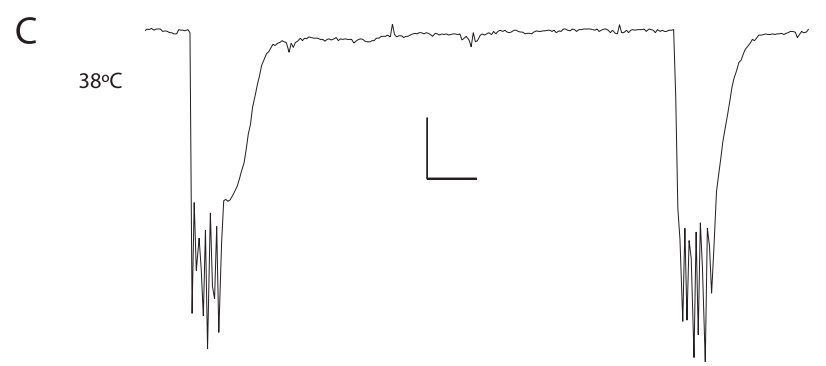

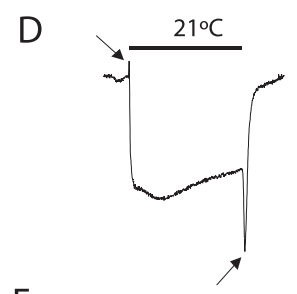

E
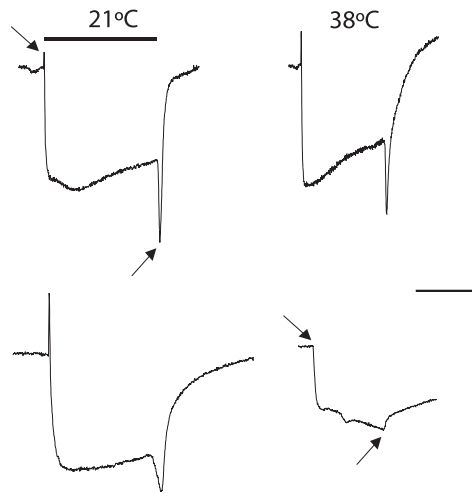

Figure 1. Electrophysiological defects in nubian animals. $A-C$, Extracellular recordings from the DLM flight pathway from controls (CS; shown in $A$ ) or nubian adults $(B, C)$ at $2-4 \mathrm{~d}$ of age. Recordings were taken at $21^{\circ} \mathrm{C}$ (top trace), at the nonpermissive $38^{\circ} \mathrm{C}$ (middle trace), and after recovery at $21^{\circ} \mathrm{C}$ (bottom trace). Control animals display no seizure activity when the temperature is raised to $38^{\circ} \mathrm{C}$. In contrast, a rapid onset of spontaneous firing is observed in nubian animals at $38^{\circ} \mathrm{C}$. After several minutes at $38^{\circ} \mathrm{C}$ the seizure activity in nubian animals ceases. Complete recovery was observed with return to the permissive temperature. $C$, An enlarged time scale trace for seizure activity in nubian at $38^{\circ} \mathrm{C}$, revealing multiple bursting muscle action potentials. Calibration: $A, B, 5 \mathrm{mV}, 0.5 \mathrm{sec} ; C, 5 \mathrm{mV}, 20 \mathrm{msec}$. D, E, Electroretinograms (ERGs) recorded from $C S$ and nubian animals. Flies were heated rapidly from 21 to $38^{\circ} \mathrm{C}$ and maintained at $38^{\circ} \mathrm{C}$ for $4 \mathrm{~min}$. Then the flies were cooled rapidly to $21^{\circ} \mathrm{C}$ and allowed to recover in darkness, with test light pulses given at regular intervals. Note the loss of on- and off-transients (arrows) in nubian flies. The on-/off-transients take $\sim 30$ min to recover when shifted back to the permissive temperature. During this recovery period nubian photoreceptors show a delayed repolarization, indicating defects in membrane excitability as well. Of each genotype $5-10$ individuals aged $2-4 \mathrm{~d}$ were tested with similar results to those shown. Calibration: $D, E, 5 \mathrm{mV}, 0.5 \mathrm{sec}$.

behavioral dysfunction, we characterized the kinetics of TS paralysis and recovery in nubian adults (Fig. 2A,B). Wild-type flies do not exhibit locomotor defects at $38^{\circ} \mathrm{C}$ for at least $30 \mathrm{~min}$. TS mutants that block action potential generation ( para $^{T S 1}$ )
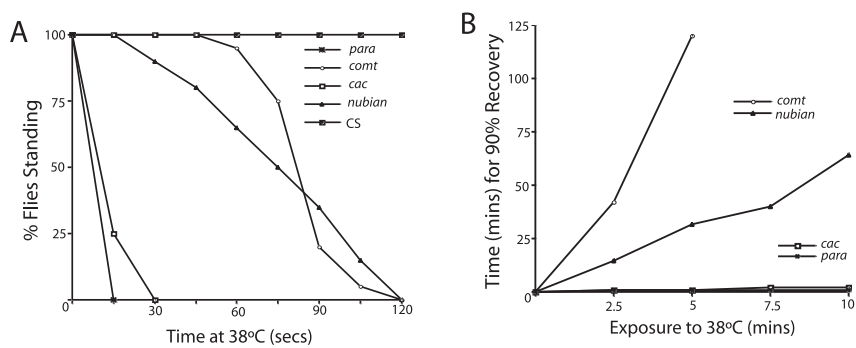

Figure 2. Temperature-sensitive nubian animals exhibit behavioral paralysis with slow recovery. $A$, The behavioral onset of motor uncoordination is plotted for nubian animals as well as for Dmca $1 A^{T S 2}$ (Kawasaki et al., 2000), para ${ }^{T S 1}$, $\mathrm{Comt}^{5 T 17}$, and CS flies. Similar to $\mathrm{Comt}^{5 T 17}$ mutants, nubian animals require exposure to $38^{\circ} \mathrm{C}$ for several minutes before the onset of paralysis. Twenty flies of the indicated genotype were aged $3-5 \mathrm{~d}$ and placed in preheated glass vials at $38^{\circ} \mathrm{C}$. The percentage of flies standing was measured in $15 \mathrm{sec}$ intervals and plotted. $B$, The recovery time after exposure to $38^{\circ} \mathrm{C}$ is plotted versus the length of exposure to the nonpermissive temperature. Unlike Dmca $A^{T S 2}$ and para ${ }^{T S 1}$ mutants that show a fast recovery, nubian mutants are affected more severely, requiring progressively longer recovery times, similar to comt ${ }^{517}$.

(Loughney et al., 1989) or synaptic transmission $\left(\mathrm{cac}^{\mathrm{NT27}}\right)$ (Reickhof et al., 2003) lead to rapid paralysis within seconds and rapid recovery when returned to the permissive temperature, regardless of the length of exposure to $38^{\circ} \mathrm{C}$. In contrast, $c o m t^{S T 17}$ and $s h{ }^{T S I}$ mutants take longer to paralyze and remain immobile for longer time periods that correlate with the length of exposure to $38^{\circ} \mathrm{C}$. Similar to $c o m t^{S T 17}$ and $s h i^{T S 1}$, nubian animals did not undergo instant paralysis but required several minutes of exposure to $38^{\circ} \mathrm{C}$ before loss of motor activity (Fig. $2 \mathrm{~A}$ ). In addition, the recovery in nubian animals was dependent on the length of exposure to $38^{\circ} \mathrm{C}$ (Fig. $2 \mathrm{~B}$ ). The behavioral phenotypes correlated with the time course of the loss and recovery of synaptic transmission measured in the visual system. We conclude that nubian mutants disrupt an essential process required for synaptic transmission and that the block is not instantaneous but, rather, requires the accumulation or depletion of an essential factor over time. This phenotype is similar to the situation found in comt $t^{S T 17}$ and $s h{ }^{T S 1}$ mutants, in which accumulation of assembled soluble $\mathrm{N}$-ethylmaleimide-sensitive factor (NSF) attachment protein (SNAP) receptor (SNARE) complexes during fusion (comt) or the depletion of synaptic vesicles (shi) eventually blocks neurotransmission. Together, the analysis of nubian adults suggests that $38^{\circ} \mathrm{C}$ induces the onset of spontaneous seizures that rapidly subside when animals are maintained at the nonpermissive temperature. After several minutes at $38^{\circ} \mathrm{C}$ the nubian mutants manifest a block of synaptic transmission as evidenced by the loss of on-/off-transients in the visual system and the behavioral paralysis. The biphasic physiological response, with seizure activity, followed by a block in synaptic transmission, argues that nubian animals may disrupt a common cellular pathway required for both membrane excitability (early phase) and synaptic transmission (late phase). Alternatively, the block in synaptic transmission preferentially may affect inhibitory GABAergic neurons earlier than excitatory neurons, leading to an imbalance in normal excitation/inhibition coupling.

To identify the gene product disrupted in the nubian mutant, we mapped the TS phenotype to $5 \mathrm{cM}$ on the left arm of the second chromosome via recombination. Deficiency mapping in the region indicated that the TS paralysis of nubian mutants was uncovered by $D f(2 L) C 144$, placing the complementation group near the synaptotagmin locus at 23A-B. This cytological interval has been studied extensively, with numerous lethal complementation groups and small deficiencies previously generated in the 
region (Littleton and Bellen, 1994). Using overlapping deficiencies (Fig. $3 A$ ), we narrowed the nubian locus to the breakpoints defined by $D f(2 L) N 19$ and $D f(2 L) N 6$, a genomic region that includes five genes and two essential loci [l(2)23Ad (Hrs) and l(2)23Ac (Littleton and Bellen, 1994)]. We ruled out the possibility that nubian mutants disrupt the Hrs locus, which functions in multivesicular body formation, via complementation with previously generated Hrs mutants (Lloyd et al., 2002). However, the TS paralysis of nubian mutants was uncovered with complementation testing with l(2)23Ac alleles, suggesting that nubian is a TS hypomorphic allele of the $l(2) 23 A c$ locus. Although the gene product encoded by the $l(2) 23 A c$ locus was unknown, previous characterization demonstrated that $l(2) 23 A c$ mutants cause lethality without obvious morphological defects in embryos (Littleton and Bellen, 1994). The four remaining candidate genes for the $l(2) 23 A c$ locus in the interval included CG3168, a predicted sugar transporter; $p g k$, encoding phosphoglycerate kinase and required for ATP generation in the terminal stage of the glycolysis pathway; CG9961, a second Drosophila homolog of $p g k$ likely generated through recent gene duplication; and CG2843, a Drosophila transcript with no homology to known genes (Fig. 3A). Given that PGK deficiency in humans has been shown to cause mental retardation, seizures, myopathy, and exercise intolerance, we predicted that nubian mutants might alter PGK function and cause TS paralysis and defects in synaptic transmission secondary to acute reductions in ATP levels in the nervous system.

To determine whether nubian mutants disrupt PGK function, we sequenced $p g k$ and CG9961 in nubian and control animals. No molecular change was identified in CG9961. In contrast, a single base pair substitution was identified in $p g k$ that caused a substitution of a charged glutamate for a valine at amino acid position 357 (Fig. 3B). Confirmation that the nubian locus disrupted PGK was obtained via TS noncomplementation with a recently identified P-element insertion in the $p g k$ locus (l(2)KG06443). This $\mathrm{P}$-element mutation is inserted at bp 932 of the ORF in exon 2 (Fig. $3 A$ ), resulting in disruption of the $p g k$ coding region and lethal noncomplementation with previously isolated $l(2) 23 A c$ alleles, confirming that the nubian mutant phenotype is attributable to disruption of PGK activity. We hereafter refer to the $n u$ bian mutant as $p g k^{\text {nubian }}$. V357 is highly conserved in all PGK homologs across evolution, suggesting that it mediates an important functional or structural role in the enzymatic activity of the protein. Mapping of the amino acid onto the crystal structure of PGK (Bernstein et al., 1997) revealed that V357E introduces a negative charge in the binding pocket for the negatively charged phosphate groups of ADP/ATP (Fig. 3C). The V357E substitution may disrupt the function of PGK constitutively by altering ADP/ATP binding. Further reductions in the function of PGK at $38^{\circ} \mathrm{C}$ likely account for the TS paralysis in $p g k^{\text {nubian }}$ mutants. PGK catalyzes the conversion of 1,3-diphosphoglycerate to 3-phosphoglycerate with the production of a single ATP molecule near the terminal step in the glycolysis pathway. It is one of two ATP-generating steps before the production of pyruvate. However, the loss/reduction of PGK function would lead to reduced pyruvate generation, effectively reducing the major substrate for ATP generation in mitochondria, thereby disrupting the citric acid cycle and electron transport chain. To test directly for ATP reduction in $p g k^{\text {nubian }}$, we performed an HPLC nucleotide analysis from head extracts of $p g k^{\text {nubian }}$ and control animals that were maintained at room temperature. The ADP/ATP ratio was found to be greatly elevated in $p g k^{\text {nubian }}$ mutants, with an approximately threefold decrease in ATP levels (Fig. 3D). We conclude that the V357E change in $p g k^{\text {nubian }}$ animals leads to a constitutive reduction in PGK function, with reduced ATP generation.

Given the reduction in ATP levels in $p g k^{\text {nubian }}$ mutants, we wanted to characterize further the behavioral and physiological consequences arising from altered ATP generation. We first analyzed the role of reduced ATP levels on lifespan. Previous studies in Drosophila have indicated that reduced caloric intake can extend lifespan greatly (Rogina et al., 2002), but the direct effects of reduced ATP levels on viability are unknown. To test lifespan, we performed viability assays on $p g k^{\text {nubian }}$ and CS control animals maintained at $28^{\circ} \mathrm{C}$. $p g k^{\text {nubian }}$ mutants showed a dramatic reduction in lifespan, with a decrease in the $\mathrm{T}_{50}$ (age at which $50 \%$ of 

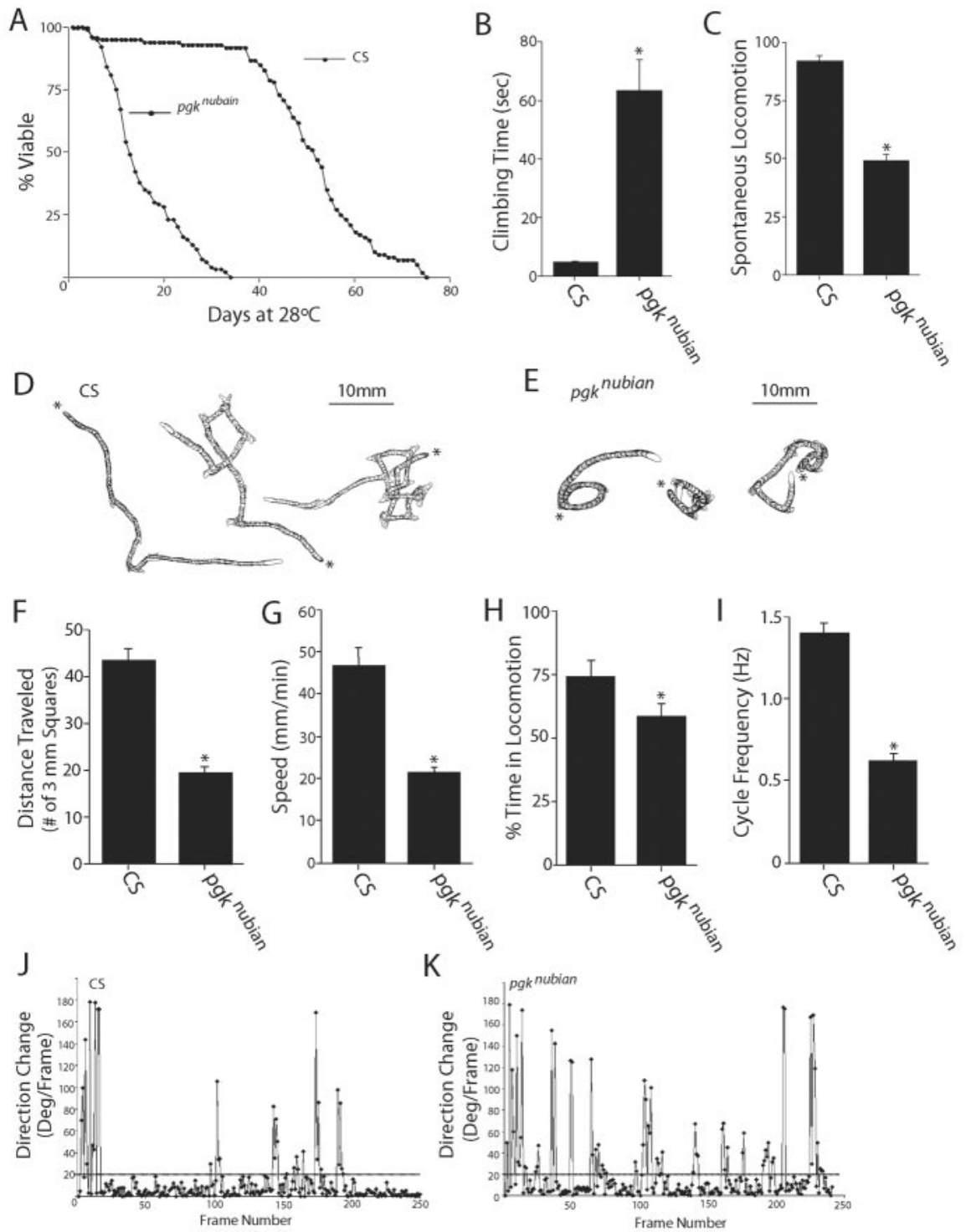

Figure 4. Behavioral analysis of $p g k^{\text {nubian }}$ animals. $A$, The $p g k^{\text {nubian }}$ mutant shows a dramatic reduction in lifespan, with a decrease in the $\mathrm{T}_{50}$ (age at which $50 \%$ of the culture has died) by $75 \%$. One hundred adult virgin males of each genotype were maintained at $28^{\circ} \mathrm{C}$, and lethality was counted daily. B, C, Behavioral assays performed on 4- to 5-d-old $p g k^{\text {nubian }}$ animals revealed a decrease in both climbing ability $(B)$ and spontaneous locomotion ( $C$. Climbing assays were performed by recording the time required for a fly placed at the bottom of a vial to $\operatorname{climb} 5 \mathrm{~cm}$, with a maximum period of $5 \mathrm{~min}$ allowed. Spontaneous locomotion was assayed by introducing a fly in a cylindrical plastic chamber (diameter, $8 \mathrm{~mm}$; height, $3 \mathrm{~mm}$ ) and quantifying the number of times it crossed a straight line bisecting the chamber in a 4 min period. The data in $B$ and Care presented as the mean \pm SEM. $D-K$ Analysis of larval locomotion with the use of computer-assisted DIAS to examine the function of the central motor pattern generator. $D, E$, Representative crawling patterns of CS and $p g k^{\text {nubian }}$ larvae. Images of the crawling larva were video recorded for a period of 2 min and digitized at 2 frames/sec; an outline or perimeter of the larva was drawn on each frame of a digital movie. Perimeters at different time points were summated to reproduce crawling patterns. The beginning of locomotion is marked by an asterisk. $F$, In a period of 2 min $p g k^{\text {nubian }}$ larvae traveled $\sim 50 \%$ less distance than that of CS larvae. Distance traveled was measured by tracing the tracks created by a crawling larva on an agarose surface during a period of 2 min and then counting the number of $3 \mathrm{~mm}$ grid squares the trace traversed. G, The speed of $p g k^{\text {nubian }}$ larvae was reduced $\sim 50 \%$ compared with control larvae. Locomotor speed was calculated by averaging the speed of the centroid (the center of the area of a perimeter) for each frame of digital movie, using the central difference method. $H, p g k^{\text {nubian }}$ mutant larvae spend $20 \%$ less time in locomotion than controls. The frame-by-frame data for direction change (shown in J, K) were used to calculate the time spent in locomotion. Periods of locomotion were defined as a crawling segment of five or more consecutive frames in which direction change was $<20^{\circ}$ for each frame when the larval images were digitized at 2 frames $/ \mathrm{sec}$. At 2 frames/sec resolution the periods of pausing were defined as frames in which the direction of change per frame was $>20^{\circ}$. I, The frequency of locomotor cycles was reduced significantly in $p g k^{\text {nubian }}$ compared with that of CS larvae. The frequency of cycles was calculated by counting directly the number of the rhythmic waves of contraction from posterior to anterior while playing back the video recording of a crawling larva and by measuring the duration in a stretch of crawling that consisted of at least five consecutive cycles. $J$, $K$, Representative plots of direction change in $p g k^{\text {nubian }}$ and CS larvae show the change of angle of the centroid for each computed frame via the central difference method. In comparisons with CS larvae the $p g k^{\text {nubian }}$ larvae have more frames with a direction change that exceeds $20^{\circ}$, indicating increased pausing compared with that for controls. ${ }^{*} p<0.05$. the culture has died) by $75 \%$, indicating a highly significant negative effect of reduced resting ATP levels on lifespan (Fig. $4 A)$. In addition to reduced lifespan, $p g k^{\text {nubian }}$ animals were more sluggish than control animals at room temperature, even shortly after pupation before any change in viability was noted. Behavioral assays performed on 4- to 5-d-old $p g k^{\text {nubian }}$ adults revealed a decrease in both climbing ability (Fig. $4 B$ ) and spontaneous locomotion (Fig. 4C), consistent with a general reduction in locomotor activity secondary to altered ATP generation. Given that ATP levels are predicted to be reduced in all tissues, these behavioral defects likely reflect dysfunction of multiple cell types within $p g k^{\text {nubian }}$ animals.

To obtain more quantitative information of the effects of reduced ATP levels on brain function, we performed larval locomotion assays to examine the central motor pattern generator (Fig. 4D-K). Drosophila larvae move by performing rhythmic waves of body wall muscle contraction and elongation that result in peristaltic propagation over a surface. A stereotypic pattern of locomotion can by achieved by allowing wandering stage third instar larva to crawl over an agarose surface in constant light. Under such conditions the larvae switch between bouts of forward peristaltic waves and episodes of head swinging, pausing, and turning. Such behavior depends on central pattern generators that organize and produce specific patterns of activation/inhibition of motor neurons independent of sensory input (Suster and Bate, 2002). We used the computerassisted DIAS motion analysis system to characterize locomotion parameters, including time spent in forward locomotion, speed, direction change, distance traveled, and cycle frequency. Representative traces from CS and $p g k^{\text {nubian }}$ mutants are shown in Figure 4, $D$ and $E$. Characteristic differences were observed in $p g k^{\text {nubian }}$ mutants, including a $>50 \%$ decrease in distance traveled (Fig. 4F) and locomotor speed (Fig. 4G). These differences in motor output were accounted for by a decrease in the time spent in locomotion (Fig. $4 H$ ) and a dramatic reduction in locomotor cycle frequency (Fig. 4I). In general, pgknubian animals spent less time in locomotion, with a much higher propensity for pauses with head swinging/turning behavior, as manifested by an increase in directional changes in pgknubian larvae (Fig. 4J,K). These behavioral abnormalities suggest that reduced ATP levels have significant effects on larval locomotion, consistent with abnormal synaptic function secondary to disruptions of PGK. 
The altered behavior of $p g k^{\text {nubian }}$ mutants could be accounted for by reductions in ATP that cause post-translational defects in neuronal function or could be secondary to large-scale misregulation of gene expression in the brain. To explore the cellular mechanisms leading to abnormal brain function in $p g k^{\text {nubian }}$ mutants, we first performed expression profiling, using Affymetrix high-density oligonucleotide GeneChip arrays. These arrays contain representative sequences for most of the 14,000 genes encoded in the Drosophila genome and provide a robust readout of transcriptional recoding in mutant animals. For our analysis we assayed male flies aged 3-4 d after eclosion that were maintained at room temperature and circadianmatched with control animals. In all, five independent arrays were performed: 3 CS and $2 p g k^{\text {nubian }}$ samples. The data were processed with the Affymetrix statistical expression algorithm, using six pairwise comparisons between mutant and wildtype arrays. For a gene to be differentially regulated, it must have been designated up- or downregulated at least twofold by the Affymetrix algorithm in $75 \%$ of the pairwise comparisons, with a $p<0.05$. Once the differentially regulated gene set was defined, we placed each gene individually through a BLAST analysis to identify similarities with known proteins or to identify known structural motifs. Finally, genes were categorized according to known or putative functions on the basis of sequence similarity. With the use of these criteria only 31 genes were upregulated, and 62 were downregulated in the $p g k^{\text {nubian }}$ mutant (supplemental Table 1, available at www. jneurosci.org), suggesting a relatively minor transcriptional response to chronic reduction in ATP levels. A majority of genes displaying altered transcription were those involved in glucose or lipid metabolism, together with cellular chaperones. Very few genes implicated directly in synaptic transmission or membrane excitability showed significant changes in mRNA level. Thus it is likely that reduced ATP levels trigger several compensation responses in metabolic pathways involved in energy metabolism, but the acute effects of reduced ATP on synaptic physiology and membrane excitability are likely secondary to post-translational defects in ATP, using proteins directly modulating neuronal activity and structure. Therefore, we analyzed synaptic structure and function at the third instar NMJ to determine how reduced ATP levels affect various synaptic parameters.

To determine the effects of reduced ATP levels on synaptic structure, we performed a quantitative analysis of synaptic growth in the $p g k^{\text {nubian }}$ mutant by visualizing anti-synaptotagmin staining as a presynaptic marker (Fig. 5). In Drosophila larvae each abdominal hemisegment (A2-A7) has a stereotyped musculature with a precise motor neuron innervation pattern. In wildtype CS third instar larvae, an average of $53 \pm 3.2( \pm$ SEM) varicosities were quantified on muscle fibers 6 and 7 (averaged over segments A3-A5). Similar to controls (Fig. 5B), pgk $k^{\text {nubian }}$ larvae displayed 54.6 $\pm 3.2( \pm$ SEM $)$ varicosities per muscle fiber $6 / 7$. Because NMJ growth varies in proportion with muscle size, we assayed synapse number in control and $p g k^{\text {nubian }}$ mutants and normalized the measurements to muscle surface area. Normalized varicosity number also was unchanged in the $p g k^{\text {nubian }} \mathrm{mu}$ tant (Fig. 5C). However, during the quantification of synapse number, obvious changes in bouton size were observed in $p g k^{\text {nubian }}$ mutants. In general, average bouton diameter was larger at $p g k^{\text {nubian }}$ synapses (Fig. $5 A$ ), especially in type $1 \mathrm{~B}$ boutons and in branching boutons with two or more branch points. To quantify this phenotype, we measured the average diameter of the five largest type $1 \mathrm{~B}$ boutons in both CS and $p g k^{\text {nubian }}$ larvae at 6/7 NMJs (Fig. 5D). Boutons were significantly larger $(p>0.001$, Student's $t$ test $)$ in $p g k^{\text {nubian }}$ animals $(5.5 \pm 0.08 \mu \mathrm{m} \pm \mathrm{SEM})$ compared with CS $(3.9 \pm 0.07 \mu \mathrm{m} \pm$ SEM $)$. Chronic reductions in ATP levels at $p g k^{\text {nubian }}$ synapses may alter cytoskeletal branching mechanisms required for bouton division, leading to larger boutons. Indeed, mutations in the microtubule-associated protein VAP-33A in Drosophila have been shown to cause increased bouton size (Pennetta et al., 2002), consistent with altered synaptic microtubule dynamics at $p g k^{\text {nubian }}$ terminals. In addition, the dissociation between synapse number and synapse size in $p g k^{\text {nubian }}$ mutants indicates that these synaptic parameters can be regulated independently. 

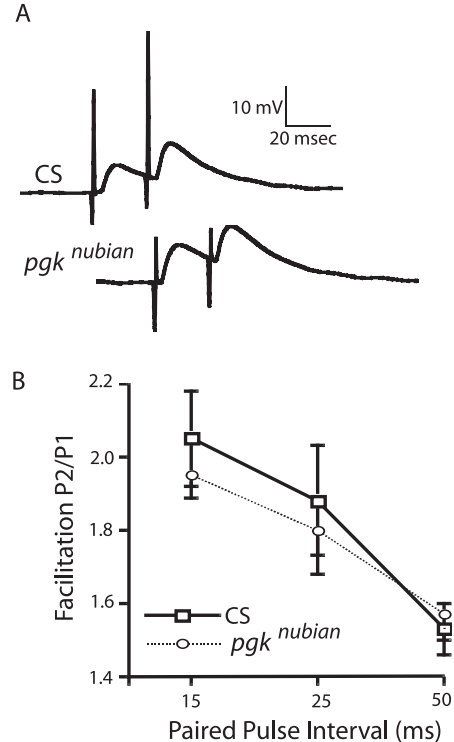

C
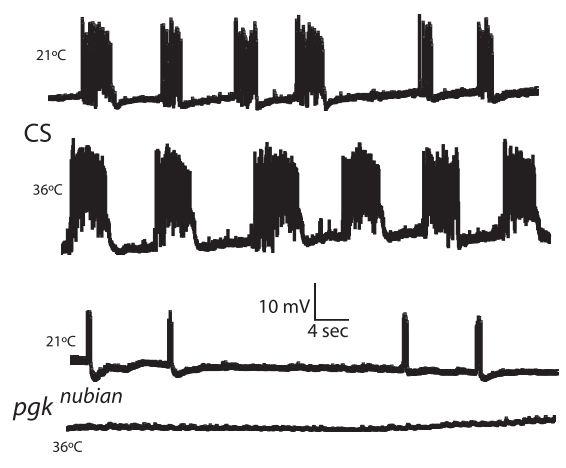

D

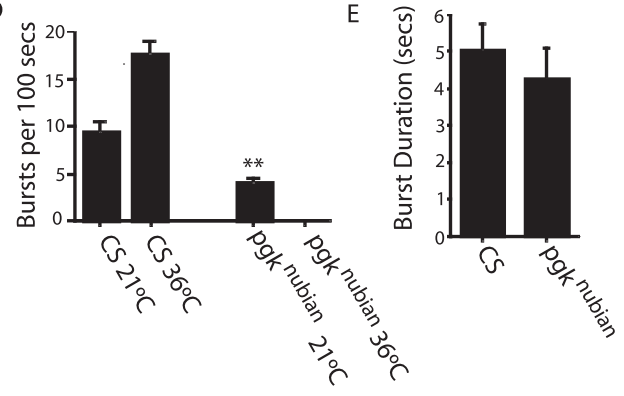

Figure 6. Paired pulse facilitation and central motor pattern generator activity in $p g k^{n u b i a n}$ mutants. A, Paired pulse facilitation in control and $p g k^{\text {nubian }}$ mutant third instar larvae was recorded in saline containing $0.2 \mathrm{~mm}$ extracellular calcium. Calibration: 10 $\mathrm{mV}, 20 \mathrm{msec}$. B, Quantification of paired pulse facilitation (amplitude of pulse 2/amplitude of pulse 1) in control and $p g k^{\text {nubian }}$ animals for 15,25 , and 50 msec intervals. No change in the amplitude of paired pulse facilitation was observed in $p g k^{\text {nubian }}$ mutants. The number of preparations analyzed was 7 CS and $5 \mathrm{pgk}^{\text {nubian }}$. C, Voltage traces of spontaneous central pattern generator activity recorded from muscle fiber 6 in third instar preparations with an intact ventral nerve cord in $1.5 \mathrm{~mm}$ extracellular calcium. In control animals spontaneous bursts of motor neuron activity were observed, with increased frequency at $36^{\circ} \mathrm{C}$. In $p g k^{\text {nubian }}$ animals spontaneous activity was reduced at room temperature and abolished at $36^{\circ} \mathrm{C}$. Calibration: $10 \mathrm{mV}, 4 \mathrm{sec}$. The number of preparations analyzed was $22 \mathrm{CS}$ at $21^{\circ} \mathrm{C}, 10 \mathrm{CS}$ at $36^{\circ} \mathrm{C}, 23 \mathrm{pg} k^{\text {nubian }}$ at $21^{\circ} \mathrm{C}$, and $22 \mathrm{pg} \mathrm{k}^{\text {nubian }}$ at $36^{\circ} \mathrm{C}$. D, Quantification of the number of bursts occurring in a $100 \mathrm{sec}$ bin in control and $p g k^{\text {nubian }}$ animals at 21 and $36^{\circ} \mathrm{C}$. pgk $k^{\text {nubian }}$ mutants have a statistically significant $\left({ }^{* *} p<0.001\right.$; Student's $t$ test) reduction in burst frequency compared with controls. At $36^{\circ} \mathrm{C}$ there was a complete absence of a central motor pattern in all $22 \mathrm{pg} k^{\text {nubian }}$ animals that were analyzed. E, Quantification of burst duration in control and $p g k^{\text {nubian }}$ animals. Although slightly reduced, there was no statistically significant change in burst duration. In general, burst duration was quite variable in both control and $p g k^{\text {nubian }}$ animals.

To determine whether the block in synaptic transmission that occurs in $p g k^{\text {nubian }}$ animals at nonpermissive temperatures is secondary to defects in membrane excitability, synaptic exocytosis, or synaptic vesicle endocytosis, we performed a quantitative analysis of synaptic physiology at the third instar NMJ. At $22^{\circ} \mathrm{C}$, evoked synaptic transmission was not altered in $p g k^{\text {nubian }}$ animals. The amplitudes of evoked excitatory junctional potentials (EJPs) and resting membrane potential were similar to controls. In addition, there was no change in paired pulse facilitation in $p g k^{\text {nubian }}$ mutants (Fig. $6 A, B$ ), indicating that short-term plasticity is intact. A slight reduction in spontaneous miniature EJP (mEJP) frequency from $1.4 \mathrm{~Hz}$ in control animals to $1.1 \mathrm{~Hz}$ in $p g k^{\text {nubian }}$ ( $p>0.001$; Student's $t$ test) was observed, suggesting a smaller recycling pool of synaptic vesicles in the mutant. To analyze the activity of the central pattern generator directly, we performed experiments on third instar larvae in which an intact ventral nerve cord with attached motor neurons was maintained. Under such conditions high-frequency bursts of neuronal activity can be detected that correspond to the underlying muscle contraction waves that mediate rhythmic locomotion. In control animals at room temperature, bursts of motor activity were observed at a frequency of $\sim 0.1 \mathrm{~Hz}$, with burst durations averaging $5 \mathrm{sec}$ (Fig. $6 C-E$ ). Consistent with the reduction in locomotor cycle frequency behaviorally observed in $p g k^{\text {nubian }}$ mutants, motor pattern bursts were reduced by one-half $(p>0.001$; Student's

$t$ test), with only a mild reduction in burst duration in $p g k^{\text {nubian }}$ mutants (Fig. 6C-E). With the shifting to $36^{\circ} \mathrm{C}$, control animals showed a twofold increase in the frequency of motor pattern bursts. In contrast, the output of the central pattern generator was abolished completely in $p g k^{\text {nubian }}$ mutants at the elevated temperatures (Fig. 6A,B), indicating an acute reduction in neuronal function accompanying the loss of PGK activity.

To quantify further the defects in synaptic transmission in $p g k^{\text {nubian }}$ mutants at $36^{\circ} \mathrm{C}$, we analyzed synaptic activity driven by nerve stimulation. Several defects were noted in $p g k^{\text {nubian }}$ mutants. First, the resting membrane potential was lower in $p g k^{\text {nubian }}$ animals than in controls at $36^{\circ} \mathrm{C}$ ( -41 compared with $-50 \mathrm{mV} ; p<0.05$; Student's $t$ test), indicating that there is an immediate effect on membrane excitability that causes muscle membrane depolarization compared with controls. It is likely that reduced function of the $\mathrm{Na}^{+} / \mathrm{K}^{+}$ATPase mediates the rapid effects of ATP depletion on excitability. In addition to changes in membrane excitability, $p g k^{\text {nubian }}$ mutants displayed a stimulationdependent reduction in evoked EJPs compared with controls (Fig. 7A,B). During 10 $\mathrm{Hz}$ nerve stimulation $p g k^{\text {nubian }}$ larvae show a gradual reduction in EJP amplitude, with a complete loss of synaptic transmission by 3 min on average. In contrast, without 10 $\mathrm{Hz}$ nerve stimulation the amplitude of evoked responses in $p g k^{\text {nubian }}$ animals showed little change (Fig. 7C). After the onset of $10 \mathrm{~Hz}$ stimulation, however, $p g k^{\text {nubian }}$ synapses underwent a rapid reduction in response similar to animals that were stimulated shortly after the temperature was shifted to $36^{\circ} \mathrm{C}$. The stimulation-dependent block in synaptic activity resembled the effects seen in $s h i^{T S 1}$ mutants (Delgado et al., 2000), in which loss of dynamin function gradually depletes synaptic vesicles from nerve terminals. Stimulation-dependent reductions in evoked responses also are seen in comt mutants (Kawasaki et al., 1998), in which loss of NSF ATPase activity blocks SNARE disassembly, leading to a reduction in free SNAREs that mediate synaptic vesicle fusion. However, in comt mutants, the amplitude of evoked responses returns to prestimulation levels with the cessation of stimulation (Kawasaki et al., 1998). This effect is not observed in $p g k^{n u b i a n}$ mutants, in which evoked responses do not recover after the cessation of stimulation. These results argue that the loss of synaptic ATP has a greater effect on the endocytosis pathway than on exocytosis itself.

To test further our hypothesis that endocytotic pathways mediated by the GTPase dynamin are more sensitive to ATP loss than NSF-dependent exocytosis at these synapses, we directly assayed SNARE complex formation in the $p g k^{\text {nubian }}$ mutant (Fig. 7D). Previous results have demonstrated that the loss of NSF function in $c o m t^{S T 17}$ mutants leads to a large increase in SNARE complex at the expense of monomeric syntaxin (Littleton et al., 1998, 2001; Tolar and Pallanck, 1998). In contrast, SNARE com- 
plexes normally found in wild-type animals are reduced in shi mutants, in which the block in vesicle endocytosis eliminates vesicle fusion and subsequent SNARE complex formation (Littleton et al., 2001). The depletion of SNARE complexes in $s h i^{T S 1}$ mutants leads to a greater proportion of syntaxin that is detected in its monomeric form (Fig. 7E). The shift of syntaxin from the SNARE complex to its monomeric form provides a robust assay for differentiating a comt-like SNARE complex accumulation phenotype from a shi-like SNARE complex depletion phenotype. The distribution of syntaxin between its monomeric and SNARE complex localization was assayed in $p g k^{\text {nubian }}$ adults after a $20 \mathrm{~min}, 38^{\circ} \mathrm{C}$ heat shock, using densitometry after blotting with anti-syntaxin antisera. In contrast to the depletion of monomeric syntaxin in comt ${ }^{S T 17}$ mutants, $p g k^{\text {nubian }}$ animals showed an accumulation of the monomeric form, similar to $s h i^{T S 1}$ (Fig. $7 F$ ). We averaged the percentage of monomeric syntaxin compared with control CS animals over many experiments in $p g k^{\text {nubian }}$, comt $^{S T 17}$, and shi $i^{T S 1}$ mutants. Whereas comt ${ }^{S T 17}$ mutants reduced the amount of monomeric syntaxin detected in brain extracts by $47 \%$ compared with CS, shi ${ }^{T S 1}$ and $p g k^{\text {nubian }}$ mutants showed increases of 36 and $65 \%$, respectively, in monomeric syntaxin compared with controls (Fig. 7E). In contrast, other synaptic proteins like synaptotagmin showed no change in signal intensity in any of the mutants. Together, the analysis of $p g k^{\text {nubian }}$ mutants provides in vivo evidence for an important role of PGK in synaptic transmission, with defects in endocytosis that occur secondary to reduced ATP levels in the nervous system.

\section{Discussion}

Glucose is the major precursor for ATP generation in neurons and is essential for maintaining normal brain function. Indeed, hypoglycemia has been demonstrated to impair many neuronal functions, including short-term memory processing (Deary et al., 2003; Sommerfield et al., 2003). Recent studies have demonstrated that glucose levels fall dramatically in the hippocampus during performance of spatial working memory tasks in rodents (McNay et al., 2000). Systemic glucose administration before testing can lead to improved memory performance, indicating that increased cognitive demand can reduce hippocampal glucose levels acutely (McNay et al., 2000). These findings also suggest that memory processing can be limited by glucose availability. Additional support for this model is provided by results indicating that both diabetic and nondiabetic patients with impaired glucose tolerance show cognitive impairments (Perlmuter et al., 1984; Richardson, 1990; Vanhanen et al., 1997). Surpris-

A CS

B
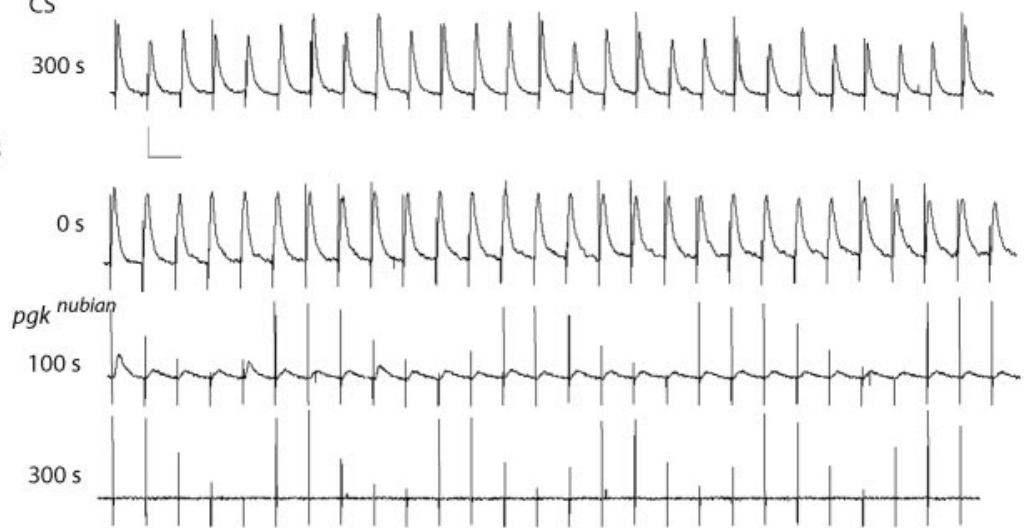

C No Prior Stimulation

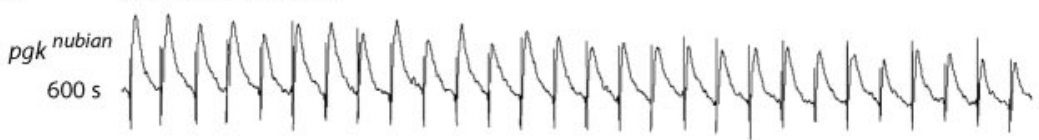

D

Figure 7. Electrophysiological defects at $p g k^{\text {nubian }}$ neuromuscular junctions. $A-C$, Voltage traces of evoked excitatory junction potentials recorded from muscle fiber 6 during $10 \mathrm{~Hz}$ stimulation at $36^{\circ} \mathrm{C}$ in either $\mathrm{CS}(A)$ or $p g k^{\text {nubian }}$ third instar larvae $(B, C)$ in 0.2 mm extracellular calcium. Calibration: $5 \mathrm{mV}, 0.1 \mathrm{sec} . p g k^{\text {nubian }}$ animals show a progressive reduction in EJP amplitude with high-frequency stimulation compared with controls. Maintaining the preparation at $36^{\circ} \mathrm{C}$ in the absence of stimulation did not result in EJP rundown. At room temperature the average initial EJP amplitude was $17.8 \pm 0.8 \mathrm{mV}(n=16)$ in CS compared with $15.5 \pm 0.7(n=20)$ in $p g k^{\text {nubian }}$, whereas resting membrane potential was $-61.43 \pm 1.4(n=16)$ in CS compared with $-57.8 \pm 1.2(n=20)$ in $p g k^{\text {nubian }}$. At $36^{\circ} \mathrm{C}$ the initial EJP amplitude was $8.8 \pm 2.1 \mathrm{mV}(n=4)$ in CS compared with $8.0 \pm 0.7$ $(n=32)$ in $p g k^{\text {nubian }}$, whereas resting membrane potential was $-49.8 \pm 2.1(n=4)$ in CS compared with $-41.8 \pm 1.4(n=32)$ in $p g k^{\text {nubian }}$.Spontaneous mEJP frequency was $1.4 \pm 0.14(n=16)$ in CS compared with $1.1 \pm 0.09(n=20)$ in pgk $k^{\text {nubian }}$. All data are presented as the mean \pm SEM. D, Model of SNARE complex (7S) generation during vesicle cycling. After vesicle fusion and SNARE complex formation the loss of NSF function in comt mutants blocks SNARE disassembly, leading to increased incorporation of monomeric syntaxin into 7S SNARE complexes. In shi mutants the vesicle recycling is blocked, leading to a reduction of SNARE complexes via NSF-mediated disassembly and an increase in monomeric syntaxin. E, Representative SNARE complex assay showing a reduction in the $7 \mathrm{~S}$ complex with an increase in monomeric syntaxin in shiflies after exposure to $38^{\circ} \mathrm{C}$, whereas comt mutants show an accumulation of $7 \mathrm{~S}$ complex (Littleton et al., 2001). The distribution of syntaxin between monomeric and SNARE complexes was analyzed from $10 \mathrm{CS}$, comt ${ }^{S T 17}$, or shi ${ }^{T S 1}$ flies after a $20 \mathrm{~min}, 38^{\circ} \mathrm{C}$ heat pulse. $F$, Western blots showing monomeric syntaxin accumulation in nubian animals compared with the depletion observed in comt ${ }^{517}$ flies. Head homogenates were prepared from $10 \mathrm{CS}, \mathrm{comt}^{\mathrm{ST17}}$, or $\mathrm{pgk}^{\text {nubian }}$ flies after a $20 \mathrm{~min}, 38^{\circ} \mathrm{C}$ heat pulse and probed for syntaxin and synaptotagmin I. G, Quantification of monomeric syntaxin levels in $\mathrm{pgk}^{\text {nubian }}$, shi ${ }^{\mathrm{TS} 1}$, and comt ${ }^{\mathrm{ST17}}$ compared with control CS. In each experiment the monomeric syntaxin levels measured by densitometry in CS were set to $100 \%$ and compared with each genotype. The number of experiments for quantification of each genotype was as follows: 4 pgk ${ }^{\text {nubian }}, 7 \mathrm{shi}^{\mathrm{TS} 1}, 10 \mathrm{comt}^{\mathrm{TT17}}$, and $10 \mathrm{CS}\left({ }^{* *} p<0.001\right.$ by Student's $t$ test analysis). ingly, quantitative serial EM reconstruction of hippocampal presynaptic terminals has revealed that $50 \%$ of synapses lack mitochondria (Shepherd and Harris, 1998), suggesting that glycolysisderived ATP may be essential for normal synaptic function. Such work has led to the current view that neurons function with limited ATP reserves and possibly even under deficit conditions, making it important to characterize neuronaldefects secondary to reduced ATP levels.

Using a new Drosophila temperature-sensitive mutant that 
disrupts PGK and subsequent ATP generation, we have tested neuronal function and development directly during both acute and chronic reductions in ATP levels. PGK is a monomeric phosphotransfer enzyme that catalyzes the conversion of 1,3diphosphoglycerate to 3-phosphoglycerate near the terminal step in the glycolysis pathway, generating a single ATP molecule during the reaction. It is one of only two cytosolic ATP-generating steps before the production of pyruvate, which then shuttles into mitochondria. In addition to its role in ATP generation during glycolysis, PGK and glyceraldehyde phosphate dehydrogenase (GAPDH) have been found to be associated with synaptic vesicles and required for glutamate uptake into vesicles (Ikemoto et al., 2003). Synaptic vesicle glutamate accumulation requires ATP to generate an electrochemical gradient, providing the driving force for neurotransmitter uptake (Maycox et al., 1988; Hell et al., 1990; Tabb et al., 1992). Ikemoto et al. (2003) have demonstrated that glycolytically produced ATP generated by GAPDH and PGK on synaptic vesicles, instead of mitochondria-derived ATP, is used for vesicular glutamate transport. A dissociation between ATP derived from glycolysis versus mitochondria also has been demonstrated in cardiac myocytes, in which glycolysis-derived ATP is used preferentially to maintain resting membrane potential (Weiss and Lamp, 1987). Similarly, fast-twitch skeletal muscle fibers use ATP produced during glycolysis, whereas slowtwitch muscle fibers use ATP synthesized by mitochondria (Holloszy and Booth, 1976). Thus in addition to global reductions in ATP levels, analysis of $p g k^{\text {nubian }}$ mutants may reveal phenotypes preferentially associated with the loss of glycolysisderived ATP.

The $p g k^{\text {nubian }}$ mutant was identified by using a behavioral screen for TS seizure mutants. $p g k^{n u b i a n}$ animals show a rapid loss of motor coordination and seizure-like activity, followed by paralysis within $2 \mathrm{~min}$ of exposure to $38^{\circ} \mathrm{C}$, unlike the instant paralysis observed in various TS ion channel mutants. $p g k^{\text {nubian }}$ mutants also take much longer to recover from a heat shock than previously described ion channel mutants, with recovery periods dependent on the length of exposure to $38^{\circ} \mathrm{C}$. Such behavioral phenotypes likely are correlated with the acute depletion of ATP levels in neurons and muscles and the time required to replenish ATP stores adequately after depletion. In addition to acute effects on neuronal function at $38^{\circ} \mathrm{C}, p g k^{\text {nubian }}$ larvae and adults show more subtle behavioral abnormalities even at room temperature, including abnormal locomotor behavior. The $p g k^{\text {nubian }}$ mutant also may prove useful for characterizing the role of ATP regulation in non-neuronal intracellular signaling. Although we have not pursued the cellular pathways that may be responsible, $p g k^{\text {nubian }}$ mutant larvae have a Tubby-like phenotype, with a shorter length and wider girth than control larvae, indicating a potential defect in organization of body shape. Further characterization of both the morphological and behavioral defects will be important to determine the cell types with altered ATP levels that are responsible for the observed phenotypes. Consistent with altered glycolysis, ATP analysis of brain extracts revealed a chronic threefold reduction in resting ATP levels, indicating that the V357E substitution in PGK constitutively disrupts enzyme activity. The V357E change introduces a negative charge in the catalytic binding pocket for ADP/ATP (Bernstein et al., 1997). With the binding of ADP and 1,3-diphosphoglycerate, a largescale conformational change occurs in the PGK hinge domain, allowing a partially assembled $\beta$-sheet to form, thereby assembling and stabilizing the catalytic active size. Such large-scale conformational changes may be particularly susceptible to mutations conferring TS dysfunction.
What are the physiological effects of altering PGK function and ATP generation in the nervous system? The chronic reductions in ATP observed at room temperature are sufficient to induce structural changes in synapse size in third instar larvae. The $p g k^{\text {nubian }}$ mutant displays a $40 \%$ increase in the diameter of the larger type $1 \mathrm{~B}$ boutons at NMJs. Surprisingly, this increase in synaptic size is not associated with a decrease in synapse number, as has been observed in vap-33A mutants that increase synapse size at the expense of synapse number (Pennetta et al., 2002). Thus bouton size and number can be regulated independently at synapses, with bouton size being more sensitive to reduced ATP. Acute reductions in ATP levels generated when $p g k^{\text {nubian }}$ animals are shifted to $38^{\circ} \mathrm{C}$ cause defects in resting membrane potential in muscles. Although the molecular targets that mediate these acute effects on membrane excitability are unknown, both the $\mathrm{Na}^{+}$/ $\mathrm{K}^{+}$-ATPase $\left(\mathrm{Na}^{+}\right.$pump) and ATP-sensitive $\mathrm{K}^{+}$channels are candidate targets for acute modulation by reduced intracellular ATP levels. The rapid and transient onset of seizures in $p g k^{\text {nubian }}$ adults also may reflect altered resting potential in neurons during acute temperature shifts. Alternatively, and perhaps more likely, defects in endocytosis in $p g k^{\text {nubian }}$ mutants preferentially may affect central GABAergic neurons that have small vesicle cycling pools, leading to a short-term disruption in the balance of excitation and inhibition in the giant fiber flight pathway. Indeed, transient seizures in the flight pathway have been observed in shibire mutants before the block in synaptic transmission (Krishnan et al., 1996), similar to the defects we have observed in $p g k^{\text {nubian }}$ mutants.

After initial changes in membrane excitability, ATP depletion results in a block in synaptic transmission. At the third instar NMJ the evoked potentials decrease rapidly with $10 \mathrm{~Hz}$ stimulation, similar to the reduction seen in TS shi mutants (Delgado et al., 2000). The loss of synaptic transmission is also activitydependent, requiring high-frequency nerve stimulation. These results suggest that the endocytotic cycle, as opposed to exocytosis, is more sensitive to reduced ATP levels. Alternatively, a reduction in glutamate transport into synaptic vesicles during recycling could account for the activity-dependent loss of neurotransmitter release. To differentiate between these two possibilities and to rule out an acute effect on the synaptic ATPase, NSF, we assayed SNARE complex formation in $p g k^{\text {nubian }}$ animals. Similar to most membrane trafficking steps, synaptic vesicle fusion requires the assembly of SNARE complexes during fusion. The integral membrane synaptic vesicle v-SNARE synaptobrevin and the plasma membrane t-SNAREs syntaxin and SNAP-25 assemble into highly stable SDS-resistant 7S SNARE complexes (Sollner et al., 1993) that can mediate fusion in reconstitution experiments (Weber et al., 1998). Native SNARE complexes also can be isolated from Drosophila head extracts. After separation on SDS-PAGE gels and Western blotting, anti-syntaxin antiserum detects both monomeric $35 \mathrm{kDa}$ syntaxin and the $73 \mathrm{kDa}$ SNARE complex containing syntaxin, synaptobrevin, and SNAP-25. In the absence of NSF, which normally dissociates the SNARE complex, one can detect a decrease in immunoreactivity of monomeric syntaxin, with a shift of syntaxin into SNARE complexes. In contrast, there is a reduction in SNARE complexes in shi mutants at the nonpermissive temperature (Littleton et al., 1998; Tolar and Pallanck, 1998). When vesicle cycling is blocked with NSF still active, SNARE complexes residing in the presynaptic membrane are disassembled, leading to a loss of SNARE complexes in shi. Thus by following the distribution of syntaxin in $p g k^{\text {nubian }}$ animals, we can determine biochemically whether neu- 
ronal SNARE complexes accumulate as in comt or are reduced as in shi. Alternatively, if PGK function is solely required for glutamate transport into vesicles, we would expect no change in the distribution of syntaxin, because fusion and recycling itself would be unaffected. Similar to shi, $p g k^{\text {nubian }}$ mutants accumulate monomeric syntaxin at the expense of the SNARE complex. Although we cannot rule out concomitant effects on synaptic vesicle glutamate transport, the accumulation of monomeric syntaxin indicates that endocytosis also is disrupted in the mutant. We conclude that reduced ATP levels have an acute effect on endocytotic pathways. Similar conclusions recently have been proposed for Drosophila TS mutants in the mitochondrial adenine nucleotide translocase, sesB (Rikhy et al., 2003). Mutations in $s e s B$ reduce mitochondrial ADP/ATP transfer and disrupt endocytosis, suggesting that a continuous mitochondrial supply of ATP also is required for presynaptic dynamin function during synaptic activity.

The $p g k^{\text {nubian }}$ mutant provides a unique experimental system to characterize further the role of altered energy metabolism on neuronal function in vivo. The disruption of ATP generation in $p g k^{\text {nubian }}$ animals is accompanied by temperature-dependent defects in resting membrane potential in muscles and an activitydependent loss of synaptic transmission in neurons. Similar results have been observed in rat neurons, in which intracellular glucose metabolism regulates acute changes in resting membrane potential and presynaptic function during neurotransmission (Shoji, 1992; Akasu et al., 1996). PGK deficiency in humans has been shown to cause mental retardation, seizures, and myopathy, depending on the severity of enzyme dysfunction (Tsujino et al., 1995). The TS behavior of $p g k^{\text {nubian }}$ animals may provide a phenotypic similarity to the exercise intolerance found in PGK deficiency patients. In conclusion, our findings in the $p g k^{\text {nubian }}$ mutant provide a potential molecular explanation for hypoglycemiainduced aberrant synaptic transmission and demonstrate important roles for glycolysis in maintaining endocytosis and synaptic transmission at presynaptic nerve terminals.

\section{References}

Akasu T, Tsurusaki M, Shoji S (1996) Depletion of glucose causes presynaptic inhibition of neuronal transmission in the rat dorsolateral septal nucleus. Synapse 24:125-134.

Andrews J, Smith M, Merakovsky J, Coulson M, Hannan F, Kelly LE (1996) The stoned locus of Drosophila melanogaster produces a dicistronic transcript and encodes two distinct polypeptides. Genetics 143:1699-1711.

Atkinson NS, Robertson GA, Ganetzky B (1991) A component of calciumactivated potassium channels encoded by the Drosophila slo locus. Science 253:551-555.

Bernstein BE, Michels PA, Hol WG (1997) Synergistic effects of substrateinduced conformational changes in phosphoglycerate kinase activation. Nature 385:275-278.

Davis GW, Bezprozvanny I (2001) Maintaining the stability of neural function: a homeostatic hypothesis. Annu Rev Physiol 63:847-869.

Deary IJ, Sommerfield AJ, McAulay V, Frier BM (2003) Moderate hypoglycaemia obliterates working memory in humans with and without insulintreated diabetes. J Neurol Neurosurg Psychiatry 74:278-279.

Delgado R, Maureira C, Oliva C, Kidokoro Y, Labarca P (2000) Size of vesicle pools, rates of mobilization, and recycling at neuromuscular synapses of a Drosophila mutant, shibire. Neuron 28:941-953.

DiMauro S, Dalakas M, Miranda AF (1983) Phosphoglycerate kinase deficiency: another cause of recurrent myoglobinuria. Ann Neurol 13:11-19.

Ganetzky B, Wu CF (1986) Neurogenetics of membrane excitability in Drosophila. Annu Rev Genet 20:13-44.

Gardiner RM (1999) Genetic basis of the human epilepsies. Epilepsy Res 36:91-95.

Hell JW, Maycox PR, Jahn R (1990) Energy dependence and functional re- constitution of the $\gamma$-aminobutyric acid carrier from synaptic vesicles. J Biol Chem 265:2111-2117.

Hoeffer CA, Sanyal S, Ramaswami M (2003) Acute induction of conserved synaptic signaling pathways in Drosophila melanogaster. J Neurosci 23:6362-6372.

Holloszy JO, Booth FW (1976) Biochemical adaptations to endurance exercise in muscle. Annu Rev Physiol 38:273-291.

Hotta Y, Benzer S (1969) Abnormal electroretinograms in visual mutants of Drosophila. Nature 222:354-356.

Ikemoto A, Bole DG, Ueda T (2003) Glycolysis and glutamate accumulation into synaptic vesicles. Role of glyceraldehyde phosphate dehydrogenase and 3-phosphoglycerate kinase. J Biol Chem 278:5929-5940.

Joiner MA, Griffith LC (1999) Mapping of the anatomical circuit of CaM kinase-dependent courtship conditioning in Drosophila. Learn Mem 6:177-192.

Katz B (1969) The release of neural transmitter substances. Liverpool, UK: Liverpool UP.

Kawasaki F, Mattiuz AM, Ordway RW (1998) Synaptic physiology and ultrastructure in comatose mutants define an in vivo role for NSF in neurotransmitter release. J Neurosci 18:10241-10249.

Kawasaki F, Felling R, Ordway RW (2000) A temperature-sensitive paralytic mutant defines a primary synaptic calcium channel in Drosophila. J Neurosci 20:4885-4889.

Koenig JH, Ikeda K (1996) Synaptic vesicles have two distinct recycling pathways. J Cell Biol 135:797-808.

Krishnan KS, Chakravarty S, Rao S, Raghuram V, Ramaswami M (1996) Alleviation of the temperature-sensitive paralytic phenotype of shibire(ts) mutants in Drosophila by sub-anesthetic concentrations of carbon dioxide. J Neurogenet 10:221-238.

Kuebler D, Zhang H, Ren X, Tanouye MA (2001) Genetic suppression of seizure susceptibility in Drosophila. J Neurophysiol 86:1211-1225.

Lewis EB, Bacher F (1968) Mutagenesis with ethyl methane-sulfonate. Dros Inf Serv 43:193.

Littleton JT, Bellen HJ (1994) Genetic and phenotypic analysis of 13 essential genes in cytological interval 22F1-2; 23B1-2 reveals novel genes required for neuronal development in Drosophila. Genetics 138:111-123.

Littleton JT, Bellen HJ, Perin M (1993) Expression of synaptotagmin in Drosophila reveals transport and localization of synaptic vesicles to the synapse. Development 118:1077-1088.

Littleton JT, Chapman ER, Kreber R, Garment MB, Carlson SD, Ganetzky B (1998) Temperature-sensitive paralytic mutations demonstrate that synaptic exocytosis requires SNARE complex assembly and disassembly. Neuron 21:401-413.

Littleton JT, Barnard RJ, Titus SA, Slind J, Chapman ER, Ganetzky B (2001) SNARE complex disassembly by NSF follows synaptic vesicle fusion. Proc Natl Acad Sci USA 98:12233-12238.

Lloyd TE, Atkinson R, Wu MN, Zhou Y, Pennetta G, Bellen HJ (2002) Hrs regulates endosome membrane invagination and tyrosine kinase receptor signaling in Drosophila. Cell 108:261-269.

Loughney K, Kreber R, Ganetzky B (1989) Molecular analysis of the para locus, a sodium channel gene in Drosophila. Cell 58:1143-1154.

Maycox PR, Deckwerth T, Hell JW, Jahn R (1988) Glutamate uptake by brain synaptic vesicles. Energy dependence of transport and functional reconstitution in proteoliposomes. J Biol Chem 263:15423-15428.

McNay EC, Fries TM, Gold PE (2000) Decreases in rat extracellular hippocampal glucose concentration associated with cognitive demand during a spatial task. Proc Natl Acad Sci USA 97:2881-2885.

Pak WL, Grossfield J, White NV (1969) Nonphototactic mutants in a study of vision in Drosophila. Nature 222:351-354.

Pennetta G, Hiesinger P, Fabian-Fine R, Meinertzhagen I, Bellen HJ (2002) Drosophila VAP-33A directs bouton formation at neuromuscular junctions in a dosage-dependent manner. Neuron 35:291-306.

Perlmuter LC, Hakami MK, Hodgson-Harrington C, Ginsberg J, Katz J, Singer DE, Nathan DM (1984) Decreased cognitive function in aging non-insulin-dependent diabetic patients. Am J Med 77:1043-1048.

Puranam RS, McNamara JO (1999) Seizure disorders in mutant mice: relevance to human epilepsies. Curr Opin Neurobiol 9:281-287.

Rao SS, Stewart BA, Rivlin PK, Vilinsky I, Watson BO, Lang C, Boulianne G, Salpeter MM, Deitcher DL (2001) Two distinct effects on neurotransmission in a temperature-sensitive SNAP-25 mutant. EMBO J 20:6761-6771.

Reickhof GE, Yoshihara M, Guan Z, Littleton JT (2003) Presynaptic N-type calcium channels regulate synaptic growth. J Biol Chem 278:41099-41108. 
Richards S, Hillman T, Stern M (1996) Mutations in the Drosophila pushover gene confer increased neuronal excitability and spontaneous synaptic vesicle fusion. Genetics 142:1215-1223.

Richardson JT (1990) Cognitive function in diabetes mellitus. Neurosci Biobehav Rev 14:385-388.

Rikhy R, Ramaswami M, Krishnan KS (2003) A temperature-sensitive allele of Drosophila sesB reveals acute functions for the mitochondrial adenine nucleotide translocase in synaptic transmission and dynamin regulation. Genetics 165:1243-1253.

Rogina B, Helfand SL, Frankel S (2002) Longevity regulation by Drosophila Rpd3 deacetylase and caloric restriction. Science 298:1745.

Saraswati S, Fox LE, Soll DR, Wu CF (2004) Tyramine and octopamine have opposite effects on the locomotion of Drosophila larvae. J Neurobiol 58:425-441.

Shepherd GM, Harris KM (1998) Three-dimensional structure and composition of $\mathrm{CA} 3 \rightarrow \mathrm{CA} 1$ axons in rat hippocampal slices: implications for presynaptic connectivity and compartmentalization. J Neurosci 18:8300-8310.

Shoji S (1992) Glucose regulation of synaptic transmission in the dorsolateral septal nucleus of the rat. Synapse 12:322-332.

Siddiqi O, Benzer S (1976) Neurophysiological defects in temperaturesensitive paralytic mutants of Drosophila melanogaster. Proc Natl Acad Sci USA 73:3253-3257.

Simmonds HA, Fairbanks LD, Morris GS, Webster DR, Harley EH (1988) Altered erythrocyte nucleotide patterns are characteristic of inherited disorders of purine or pyrimidine metabolism. Clin Chim Acta 171:197-210.

Soll DR (1995) The use of computers in understanding how animal cells crawl. Int Rev Cytol 163:43-103.

Sollner T, Whiteheart SW, Brunner M, Erdjument-Bromage H, Geromanos S, Tempst P, Rothman JE (1993) SNAP receptors implicated in vesicle targeting and fusion. Nature 362:318 -324.

Sommerfield AJ, Deary IJ, McAulay V, Frier BM (2003) Moderate hypoglycemia impairs multiple memory functions in healthy adults. Neuropsychology 17:125-132.

Stewart BA, Atwood HL, Renger JJ, Wang J, Wu CF (1994) Improved stability of Drosophila larval neuromuscular preparations in haemolymphlike physiological solutions. J Comp Physiol [A] 175:179-191.

Suster ML, Bate M (2002) Embryonic assembly of a central pattern generator without sensory input. Nature 416:174-178.
Suzuki DT, Grigliatti T, Williamson R (1971) Temperature-sensitive mutations in Drosophila melanogaster. VII. A mutation ( para $^{t s}$ ) causing reversible adult paralysis. Proc Natl Acad Sci USA 68:890-893.

Tabb JS, Kish PE, Van Dyke R, Ueda T (1992) Glutamate transport into synaptic vesicles. Roles of membrane potential, $\mathrm{pH}$ gradient, and intravesicular pH. J Biol Chem 267:15412-15418.

Tein I (1996) Metabolic myopathies. Semin Pediatr Neurol 3:59-98.

Titus SA, Warmke JW, Ganetzky B (1997) The Drosophila erg K ${ }^{+}$channel polypeptide is encoded by the seizure locus. J Neurosci 17:875-881.

Tolar LA, Pallanck L (1998) NSF function in neurotransmitter release involves rearrangement of the SNARE complex downstream of synaptic vesicle docking. J Neurosci 18:10250-10256.

Tsujino S, Shanske S, DiMauro S (1995) Molecular genetic heterogeneity of phosphoglycerate kinase (PGK) deficiency. Muscle Nerve 3:S45-S49.

Turrigiano GG (1999) Homeostatic plasticity in neuronal networks: the more things change, the more they stay the same. Trends Neurosci 22:221-227.

Vanhanen M, Koivisto K, Karjalainen L, Helkala EL, Laakso M, Soininen H, Riekkinen P (1997) Risk for non-insulin-dependent diabetes in the normoglycaemic elderly is associated with impaired cognitive function. NeuroReport 8:1527-1530.

Wang JW, Sylwester AW, Reed D, Wu DA, Soll DR, Wu CF (1997) Morphometric description of the wandering behavior in Drosophila larvae: aberrant locomotion in $\mathrm{Na}^{+}$and $\mathrm{K}^{+}$channel mutants revealed by computer-assisted motion analysis. J Neurogenet 11:231-254.

Wang JW, Soll DR, Wu CF (2002) Morphometric description of the wandering behavior in Drosophila larvae: a phenotypic analysis of $\mathrm{K}^{+}$channel mutants. J Neurogenet 16:45-63.

Weber T, Zemelman BV, McNew JA, Westermann B, Gmachl M, Parlati F, Söllner TH, Rothman JE (1998) SNAREpins: minimal machinery for membrane fusion. Cell 92:759-772.

Weiss JN, Lamp ST (1987) Glycolysis preferentially inhibits ATP-sensitive $\mathrm{K}^{+}$channels in isolated guinea pig cardiac myocytes. Science 238:67-69.

Yuan LL, Ganetzky B (1999) A glial-neuronal signaling pathway revealed by mutations in a neurexin-related protein. Science 283:1343-1345.

Zinsmaier KE, Eberle KK, Buchner E, Walter N, Benzer S (1994) Paralysis and early death in cysteine string protein mutants of Drosophila. Science 263:977-980. 